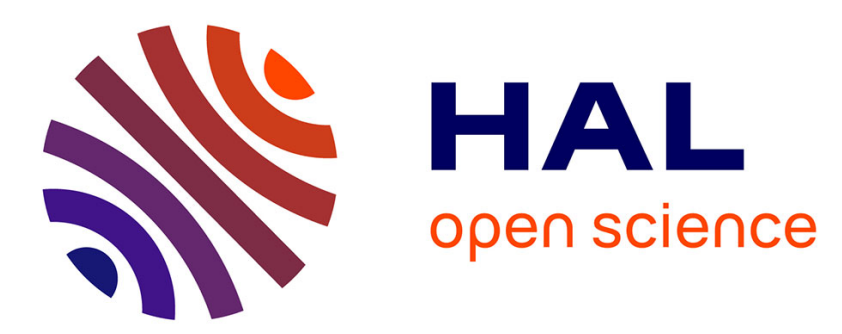

\title{
Charge carriers transport properties in CdTe measured with time of flight technique
}

\author{
G. Ottaviani
}

\section{To cite this version:}

G. Ottaviani. Charge carriers transport properties in CdTe measured with time of flight technique. Revue de Physique Appliquée, 1977, 12 (2), pp.249-254. 10.1051/rphysap:01977001202024900 . jpa00244153

\section{HAL Id: jpa-00244153 https://hal.science/jpa-00244153}

Submitted on 1 Jan 1977

HAL is a multi-disciplinary open access archive for the deposit and dissemination of scientific research documents, whether they are published or not. The documents may come from teaching and research institutions in France or abroad, or from public or private research centers.
L'archive ouverte pluridisciplinaire HAL, est destinée au dépôt et à la diffusion de documents scientifiques de niveau recherche, publiés ou non, émanant des établissements d'enseignement et de recherche français ou étrangers, des laboratoires publics ou privés. 


\title{
CHARGE CARRIERS TRANSPORT PROPERTIES IN CdTe MEASURED WITH TIME OF FLIGHT TECHNIQUE
}

\author{
G. OTTAVIANI
}

University of Modena, Italy

\begin{abstract}
Résumé. - On passe en revue les résultats expérimentaux obtenus par la méthode du temps de vol sur les paramètres de transport dans le tellurure de cadmium de haute résistivité. On s'intéresse tout d'abord aux mesures effectuées sur les cristaux compensés à l'indium et au chlore, puis on analysera l'influence des centres de diffusion chargés sur la mobilité des électrons et des trous. Finalement, on comparera les résultats expérimentaux à ceux prévus théoriquement.
\end{abstract}

\begin{abstract}
The experimental results of charge carrier transport properties obtained in high resistivity CdTe with time of flight technique is reviewed. The data for electrons and holes measured in $\mathrm{Cl}$ and In doped material are presented. The effect of ionized scattering centers are also analysed. A comparison among theory and experiment is made.
\end{abstract}

1. Introduction. - Cadmium telluride is a rather promising semiconductor material having potential applications in many fields. It is, at present used mainly as a nuclear detector working at room temperature. However the performance of the devices are currently limited by charge carrier trapping effects, due to the presence of imperfections (or impurity) in the crystal. The main purpose of this paper is to review the experimental data on charge carrier transport properties obtained in high resistivity material by the time of flight technique (TOF). The TOF technique utilizes the same kind of material and the same experimental configuration used for a nuclear detector.

Section 2 contains a theoretical analysis performed with the Monte Carlo technique on carrier transport including also ionized impurity scattering. A short analysis on trapping-detrapping phenomena is also given. Section 3 contains a set of experimental data both on transport properties and on trappingdetrapping effects.

2. Theoretical analysis. -2.1 CARRIER DRIFT velOCITY. - A numerical Monte Carlo technique has been used to solve the transport equations [1] ; the validity of such calculations is limited only by the theoretical model used to describe the problem. Such a method enables us to obtain a better understanding of the relevant microscopic phenomena which occur in $\mathrm{CdTe}$, a material which exhibits negative differential mobility (Gunn effect).

The conduction band has been idealized by a central valley and 4 satellite valleys equivalent to each other and raised by an amount of energy $1.5 \mathrm{eV}$ with respect to the central one. The scattering mechanisms which have been taken into account are polar optical, deformation potential acoustic, and ionized impurity in both central and satellite valleys. Furthermore, non equivalent and equivalent intervalley scattering mechanims have been considered. In particular the effect of ionized impurity scattering has been included. The details of calculation have been given in ref. [1].

In figure $1 v_{\mathrm{d}}$ vs. $E$ characteristics obtained from the Monte Carlo calculations are shown for two different

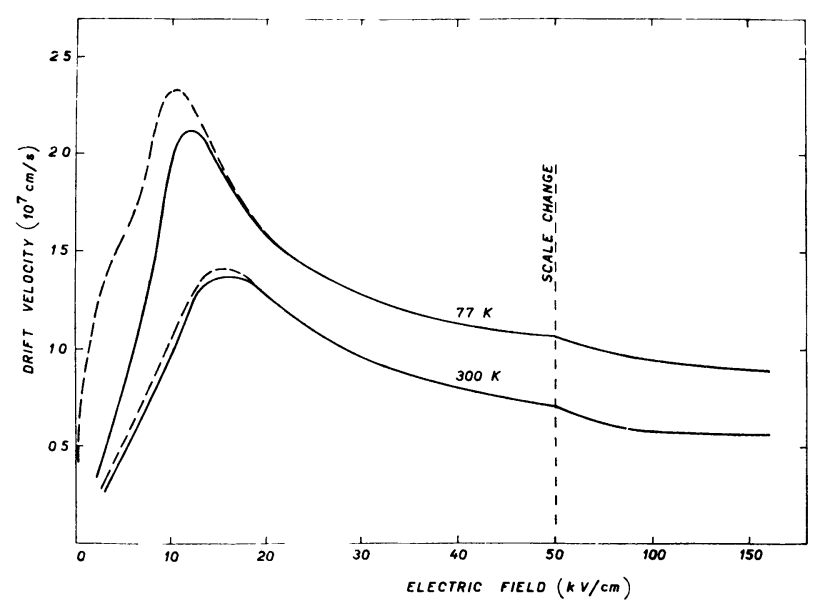

FIG. 1. - Drift velocity of electrons as a function of field strength with zero (dashed lines) and $10^{17} \mathrm{~cm}^{-3}$, (full lines) impurity content.

values of lattice temperature and impurity content. In order to discuss these curves let us first point out that energy equilibrium is maintained at low fields by polar scattering with optical phonons. At a critical field this scattering, as is well known [2], is no longer able to fully dissipate the energy gained by the electrons from the field, and electric breakdown would occur (polar runaway) if a mechanism (intervalley scattering in our 
case) did not become active. The values obtained for this runaway field with the standard parameters are about $9 \mathrm{kV} / \mathrm{cm}$ at $77 \mathrm{~K}$ and $12 \mathrm{kV} / \mathrm{cm}$ at $300 \mathrm{~K}$.

From the analysis of figure 2 it is possible to verify that the above description does represent the physical situation determined by the present model. Figure 2

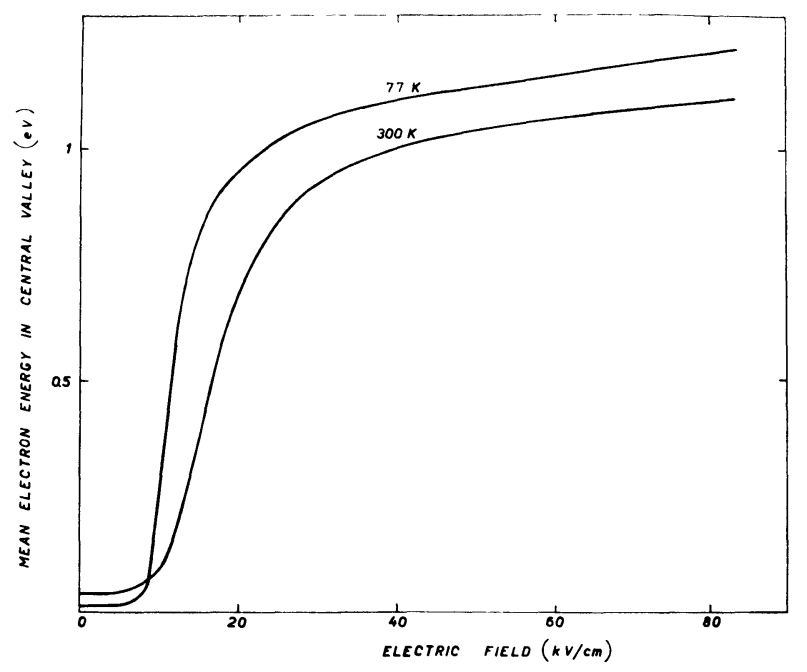

Fig. 2. - Mean electron energy in the central valley as a function of field strength with $10^{17} \mathrm{~cm}^{-3}$ impurity content.

shows the mean electron energy in the central valley as a function of the applied electric field for the two values of temperature considered above.

In figure 1 the curve at $77 \mathrm{~K}$ with zero impurity content has a peculiar shape at fields below threshold, where its slope first reduces before rising again. In fact, $v_{\mathrm{d}}$ takes on subohmic values as soon as the mean electron energy increases and more electrons can undergo polar optical scattering ; this feature is predicted for dominant polar optical mode at lattice temperatures lower than the optical phonon temperature $\theta_{0}(248 \mathrm{~K})$. At higher filed strengths, at which the electron mean energy is increasing above $\theta_{0}$, polar optical scattering becomes less effective in randomizing the electron momenta and $v_{\mathrm{d}}$ rises more steeply. At $300 \mathrm{~K}$, on the contrary, $\theta_{0}$ is lower than the lattice temperature and polar optical scattering alone would produce weakly superohmic values of $v_{d}[3]$; this effect has almost disappeared in the present calculation as a consequence of acoustic phonon scattering.

A striking feature of the curves in figure 1 is that the effect of ionized impurity scattering on the drift velocity is still appreciable (and strong at $77 \mathrm{~K}$ ) at electric fields around the threshold. At such fields the mean energy of the electrons in our calculations is already several tenths of $\mathrm{eV}$, and one would expect the Coulomb scattering by ionized impurities to be very weak, thus having a negligible effect on electric conduction. Let us at this point make it clear that this high field effect of ionized impurity scattering must not be attributed to an influence of the Coulomb scattering on very fast electrons, as was verified directly in the Monte Carlo simulation by artificially requiring that impurities were ineffective for electron energies above $0.1 \mathrm{eV}$. In this case, in fact, we obtained almost the same results as when impurities were effective at all energies. Thus, in order to interpret this effect correctly, we examined how the electrons contributed to the drift velocity as a function of their energy. Figure 3 shows this energy

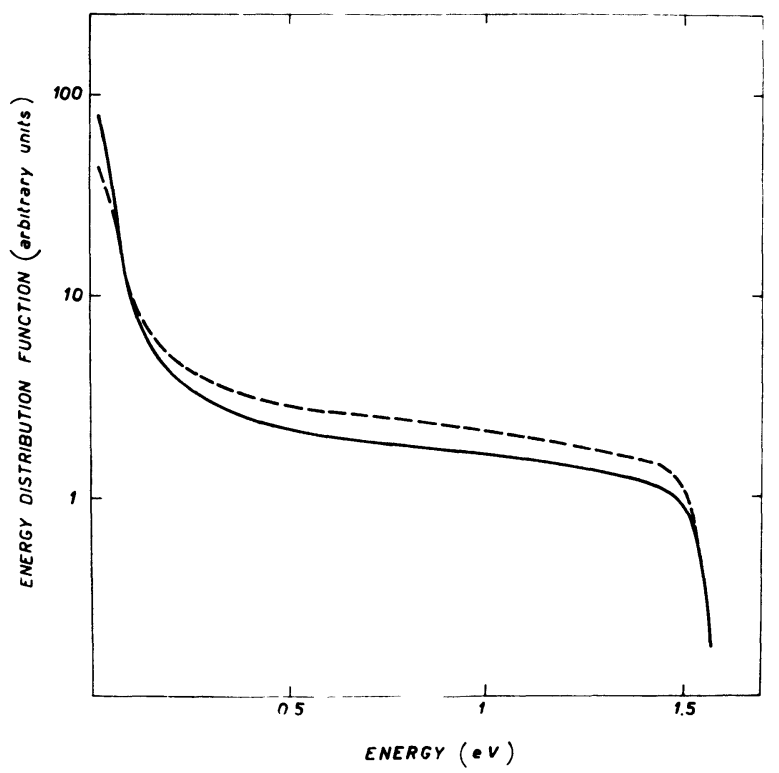

FIG. 3. - Energy distribution function of electrons in the central valley at $77 \mathrm{~K}$ for an electric field of $10.5 \mathrm{kV} / \mathrm{cm}$ with zero (dashed lines) and $10^{17} \mathrm{~cm}^{-3}$ (full line) impurity content.

distribution function with and without impurities at $77 \mathrm{~K}$ and $10.5 \mathrm{kV} / \mathrm{cm}$ field strength. The randomizing effect of ionized impurity scattering is such as to hold an appreciably larger fraction of electrons in the range of low energies. It is possible to conclude that ionized impurity scattering lowers the total drift velocity even at high fields by acting only on the low energy electrons ; more precisely by keeping a large number of them in the low energy range, which corresponds to a low mobility region of the distribution function.

A theoretical analysis of holes drift velocity performed with the Monte Carlo technique has given the result shown in figures 4 and 5. The details of such calculations are given in refs. [4] and [5]. We simply mention that interband and intraband transitions due to polar optical phonons have been taken into account, using the correct form of the degenerate valence band and the correct symmetry of the hole wave functions. It can be seen that the heating of the holes at fields $E<30 \mathrm{kV} / \mathrm{cm}$ and temperatures $<100 \mathrm{~K}$ is moderate, much less than in the case of electrons.

2.2 TrapPING-DETRAPPING EFFECTS. - The carriers, during their motion inside the semiconductor material, can be trapped by impurities in the crystal [6]. After spending some time in the center these trapped 


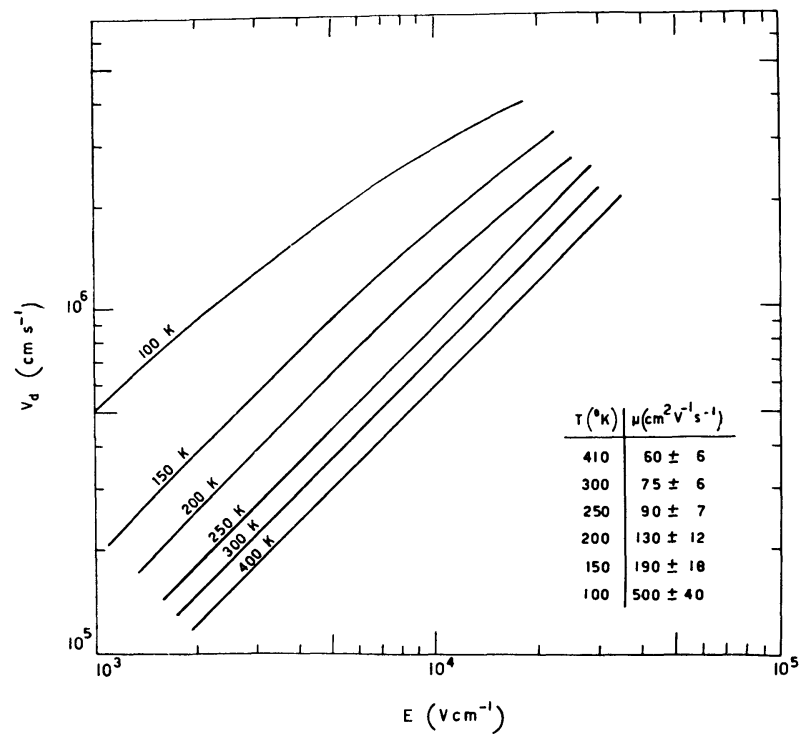

Fig. 4. - Drift velocity of the holes as a function of the electric field at different temperatures obtained with Monte Carlo calculations.

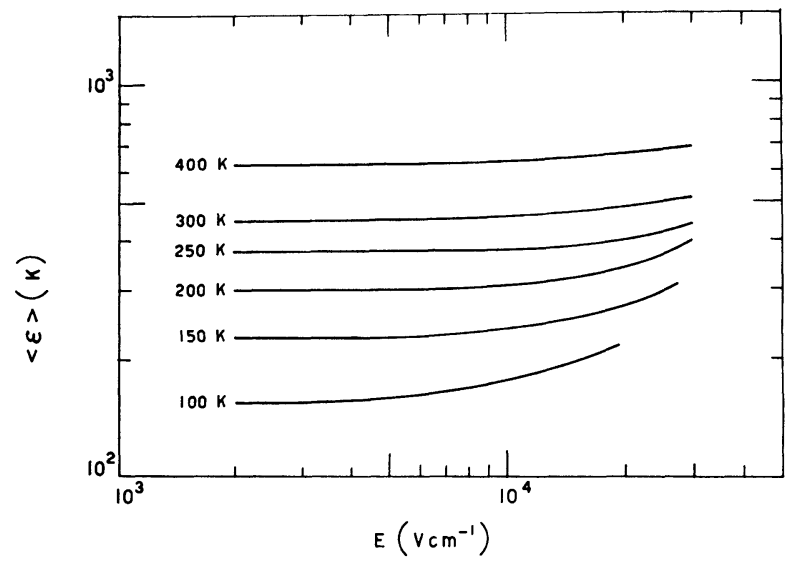

Fig. 5. - Mean energy of the holes as a function of the applied electric field at different temperatures.

carriers can be re-emitted [6] into conduction (or valence) band. Such phenomena can be described by two time constants : the mean free drift time $\tau^{+}$and the detrapping time $\tau_{\mathbf{D}}$.

The increase of the electric field $\mathrm{E}$ can influence $\tau^{+}$ essentially in two ways. One is a reduction of the capture cross section due to the reduction of the potential barrier associated to the trapping center (Poole-Frenkel effect) [6, 7]. This mechanism causes an increase of $\tau^{+}$. The second mechanism which causes an increase of $\tau^{+}$is the well known heating effect of the carriers by the electric field. According to the Lax theory [8] $\tau^{+}$should increases proportionally to $\left(T_{\mathrm{L}} / T_{\mathrm{e}}\right)^{-2}$ where $T_{\mathrm{L}}$ is the lattice-temperature and $T_{\mathrm{e}}$ is the carrier temperature assuming a maxwellian distribution function.

The detrapping probability can be enhanced by increasing the electric field in two ways, depending upon the activation energy of the trap and the magnitude of the applied electric field. The first mechanism is the same Poole-Frenkel effect previously described which leads to a decrease of the trap potential barrier by a quantity proportional to $E^{1 / 2}$ [8]. The second mechanism which generally occurs at electric field much higher than the other is the tunnel effect which leads to a temperature independent re-emission process [9-11]. These effects are schematically shown in figure 6.

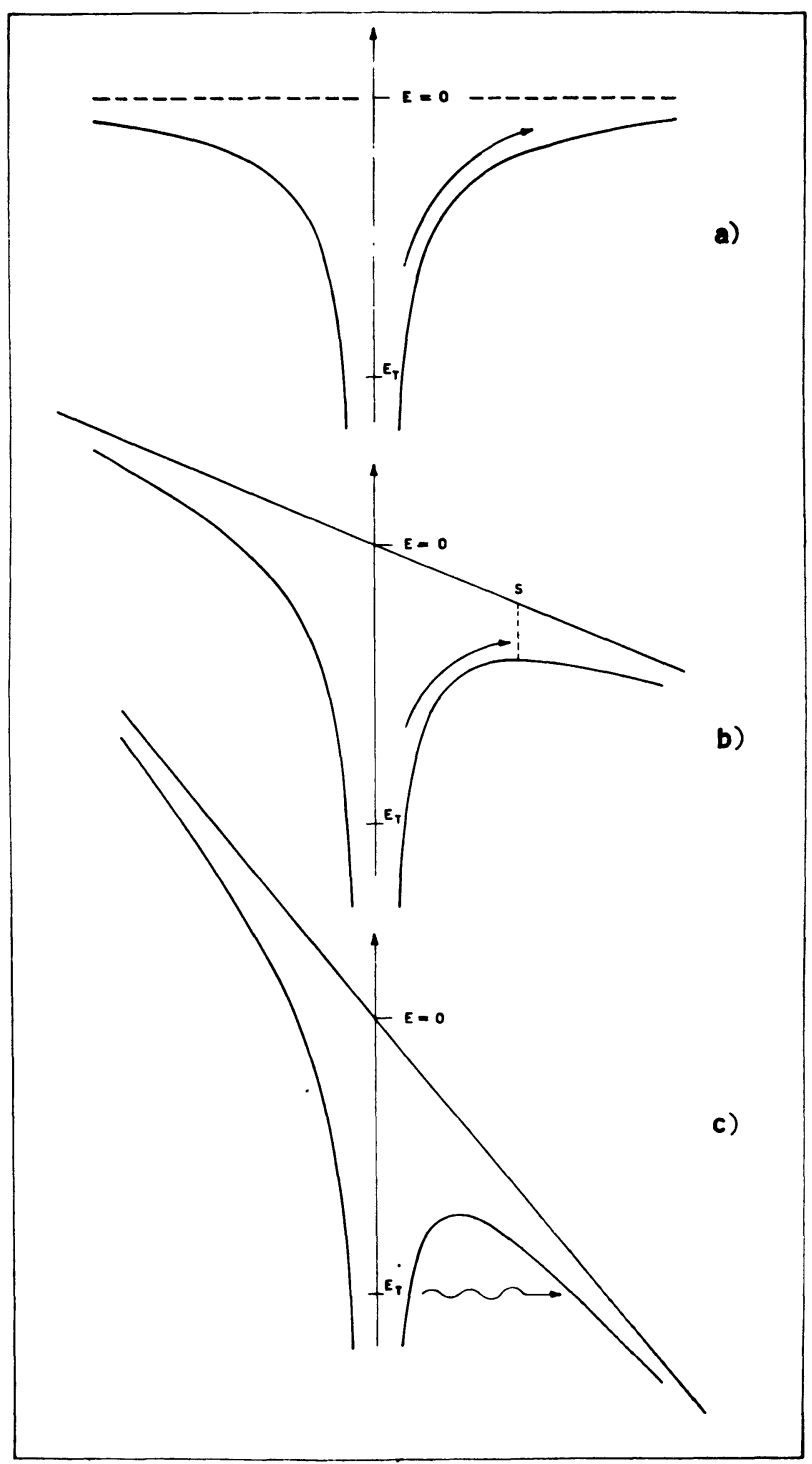

FIG. 6. - Schematic diagram of the effect of the electric field on a coulombic scattering center. By increasing the electric field the coulombic barrier is lowered going from Poole Frenkel effect to tunnel effect.

3. Experimental results. - The measurements were performed in high resistivity $\mathrm{Cl}$ or In compensated material using the time of flight technique. Details of the experimental apparatus and of the analysis has been given elsewhere [12, 13].

A complete set of electron drift velocity as a function 
of electric field and temperature obtained in the purest avalaible material is shown in figure 7 [12]. These data are in good agreement with the theoretical expectation in the case of $10^{17} \mathrm{~cm}^{-3}$ impurity scattering centers are present. By increasing the doping concentration, the characteristics of the material change [14].

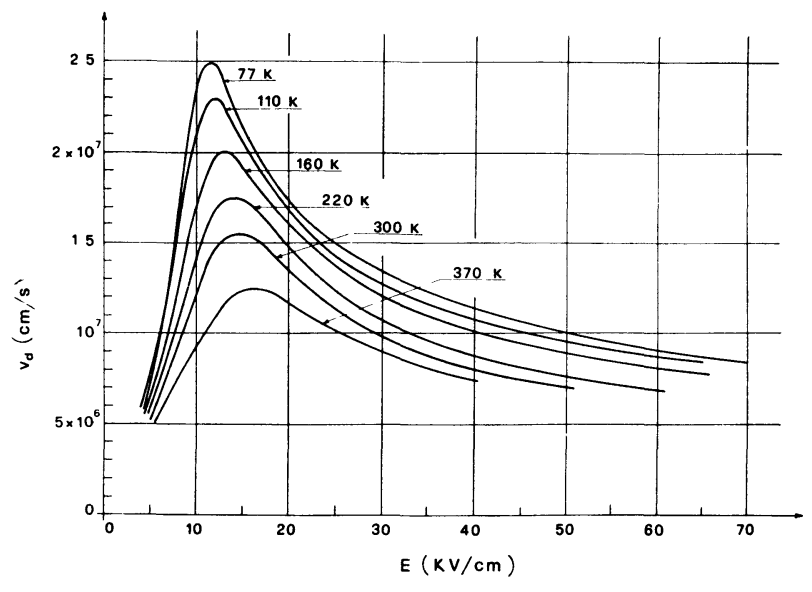

Fig. 7. - Experimental values of electron drift velocity in compensated CdTe.

The dependence of electron drift velocity upon electric field in the lightly doped material (A) and the heavily doped material (B) at 300 and $80 \mathrm{~K}$ are shown in figures 8 and 9 .

Theoretical calculations [1] of the drift velocity characteristic without ionized scattering centers (solid line) and for $10^{17} \mathrm{~cm}^{-3}$ of such centers (dashed line) are included. At $300 \mathrm{~K}$ the effect of the scattering on the drift velocity is negligible even for $10^{17} \mathrm{~cm}^{-3}$ centers. At $80 \mathrm{~K}$ the data from material $\mathrm{A}$ can be fitted with

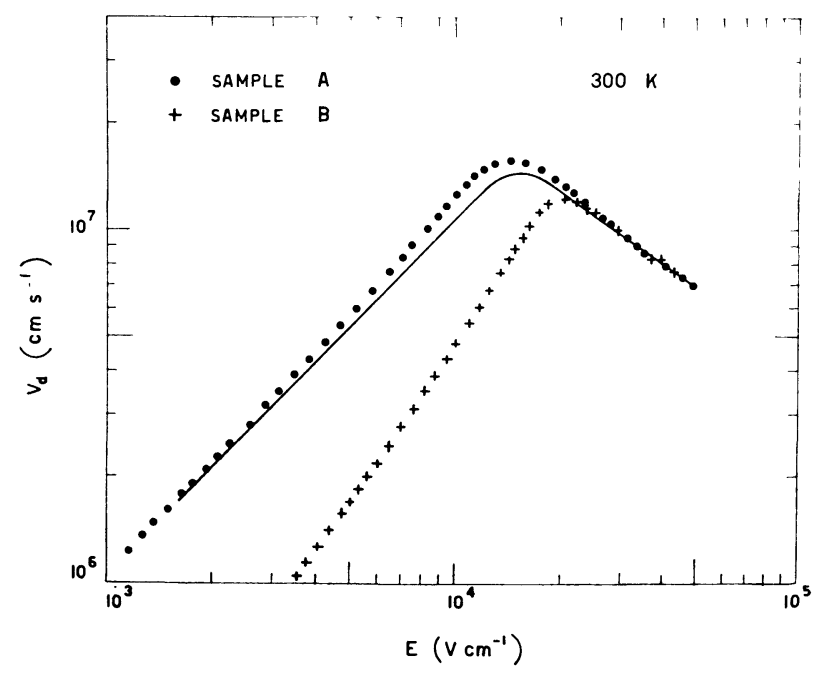

FIG. 8. - Electron drift velocity at room temperatures, as a function of the electric field in two different materials, containg approximately $1 \mathrm{ppm}$ ( $\square$ sample A) and $100 \mathrm{ppm}$ (+ sample B) of indium. The solid and dashed lines represent theoretical data for respectively zero and $10^{17} \mathrm{~cm}^{-3}$ ionized centers. At this temperature the effect of the $10^{17} \mathrm{~cm}^{-3}$ scattering centers in the drift velocity is negligible.

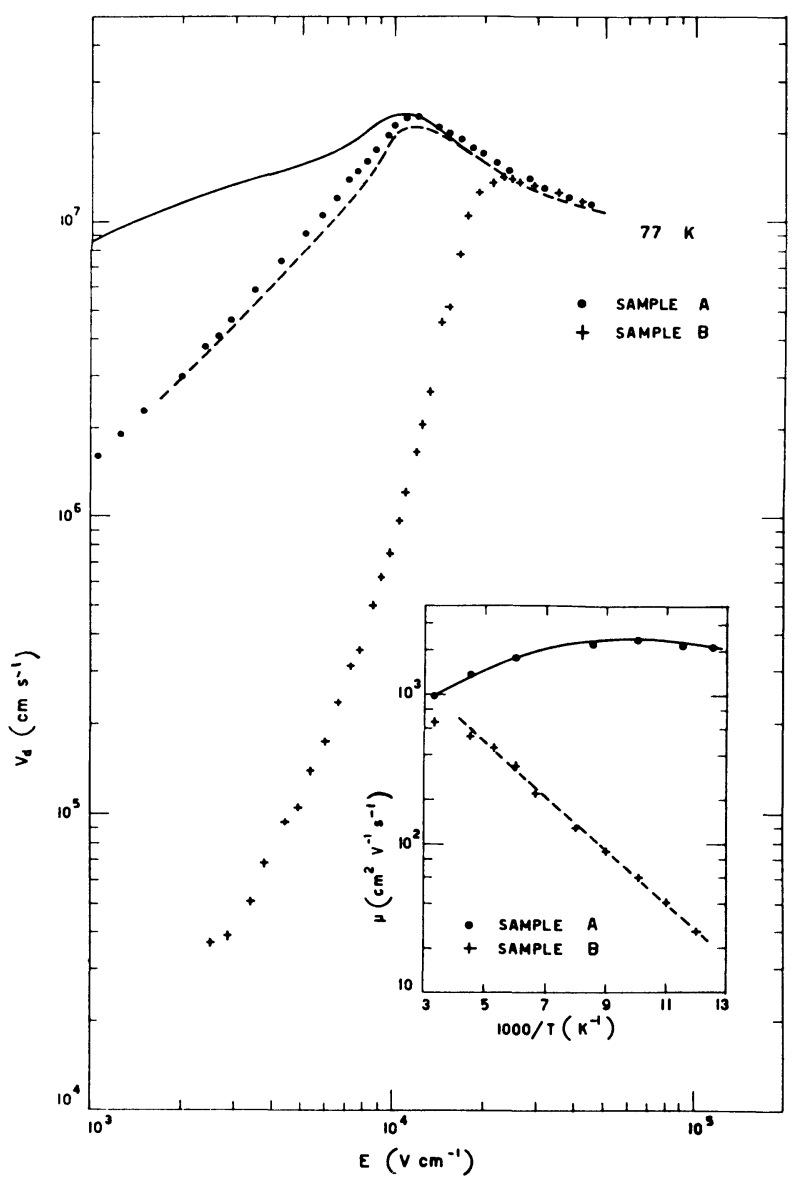

FIG. 9. - Electron drift velocity at $80 \mathrm{~K}$ as a function of the electric field in the same two materials. The solid and dashed lines represent theoretical data for respectively zero and $10^{17} \mathrm{~cm}^{-3}$ ionized centers. The insert shows the drift mobility measured at $2 \times 10^{2} \mathrm{~V} / \mathrm{cm}$ versus $1000 / \mathrm{T}$ for material containg approximately $1 \mathrm{ppm}$ ( $\mathrm{ample} \mathrm{A}$ ) and $100 \mathrm{ppm}$ (+ sample B) of indium. The curved line $\left(-\_-\right)$represents the calculated values of the electron mobility obtained from

$$
\mu=\left(1 / \mu_{\mathrm{I}}+1 / \mu_{1}\right)^{-1}
$$

where $\mu_{\mathrm{I}}$ is the mobility calculated from the Conwell Weisskopf formula for $10^{17} \mathrm{~cm}^{-3}$ singly ionized centers and $\mu_{1}$ is the lattice limited mobility taken from Segall et al. A straight line was drawn through the data for sample B to indicate the linearity of the data and determine the activation energy of the trap.

theoretical results if the Coulombic scattering by $10^{17} \mathrm{~cm}^{-3}$ centers is considered. The values of Ohmic mobility measured at $2000 \mathrm{~V} / \mathrm{cm}$ in material $A$ are shown in the insert of figure 9 as a function of $1000 / T$ and are in good agreement with the Conwell and Weisskopf theory [9] assuming $1.5 \times 10^{17} \mathrm{~cm}^{-3}$ ionized centers.

It is clear, specially at $80 \mathrm{~K}$, that scattering by ionized centers maintains its influence on the electron drift velocity up to very high fields, i. e., above the threshold value (around $11000 \mathrm{~V} / \mathrm{cm}$ at $80 \mathrm{~K}$ ). As stated before, it has been shown [1] that this phenomenum must not be attributed to the interaction of the ionized centers with very fast electrons but rather to the large number of electrons that the scattering process 
keeps in the more randomized low energy part of the distribution function where the scattering mechanisms are more effective.

On the contrary, it is impossible to explain the data of the heavily doped material B by considering only Coulombic scattering. In fact the linear dependence of $\ln \mu$ versus $1000 / \mathrm{T}$ measured at $2000 \mathrm{~V} / \mathrm{cm}$ shown in figure 9 indicates that the experimental data have an exponential and not a power law dependence upon the temperature. An analysis of the experimental data obtained in material $B$ indicates that mobility is reduced by trapping-detrapping phenomena and that these are influenced by the electric field through the Poole-Frenkel and tunneling effects. These two effects act at the same time and in order to separate them we have divided the examined temperature and electric field range into two regions : a low electric field range in which Poole-Frenkel effect is dominant, and a high electric field range for temperatures $T<103 \mathrm{~K}$ in which tunneling is effective. The results of the analysis are shown in figures 10 and 11 where the data are interpreted with Poole Frenkel effect (Fig. 10) and tunnel (Fig. 11) at low and high field respectively.

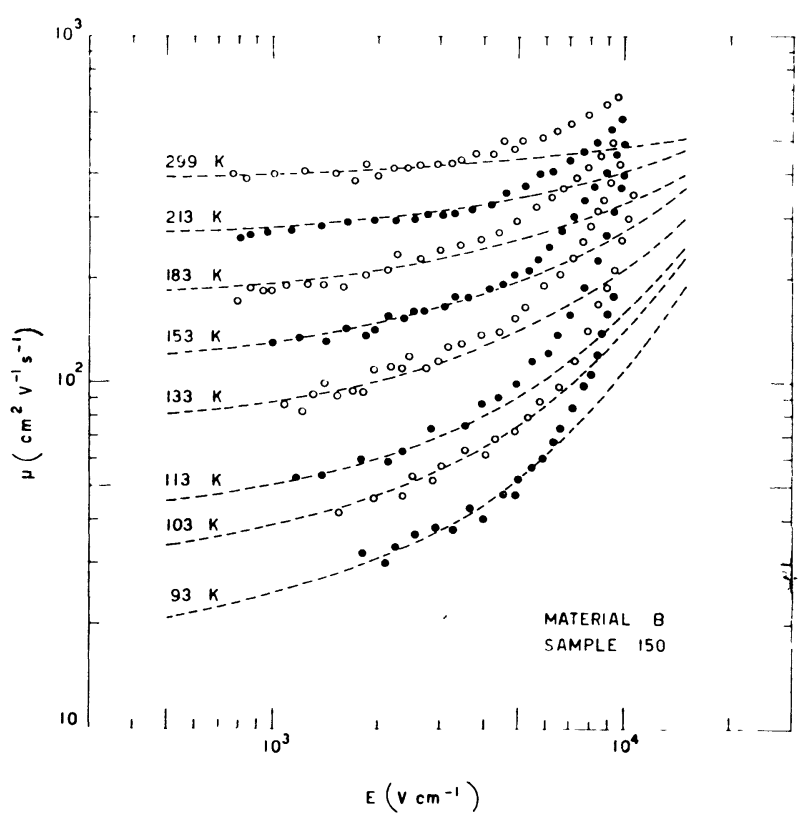

Fig. 10. - Electron drift mobility as a function of the electric field at different temperatures in B type materials. Dashed lines show the result of the Poole-Frenkel analysis.

The hole mobility is strongly affected by trapping and detrapping effects [5]. A typical set of data obtained in high resistivity material is shown in figure 12 as a function of temperature and electric field. These data which represent the situation up to date show :

a) At a fixed electric field the mobility decreases with decreasing temperature.

b) At a fixed temperature the mobility increases with increasing electric field.

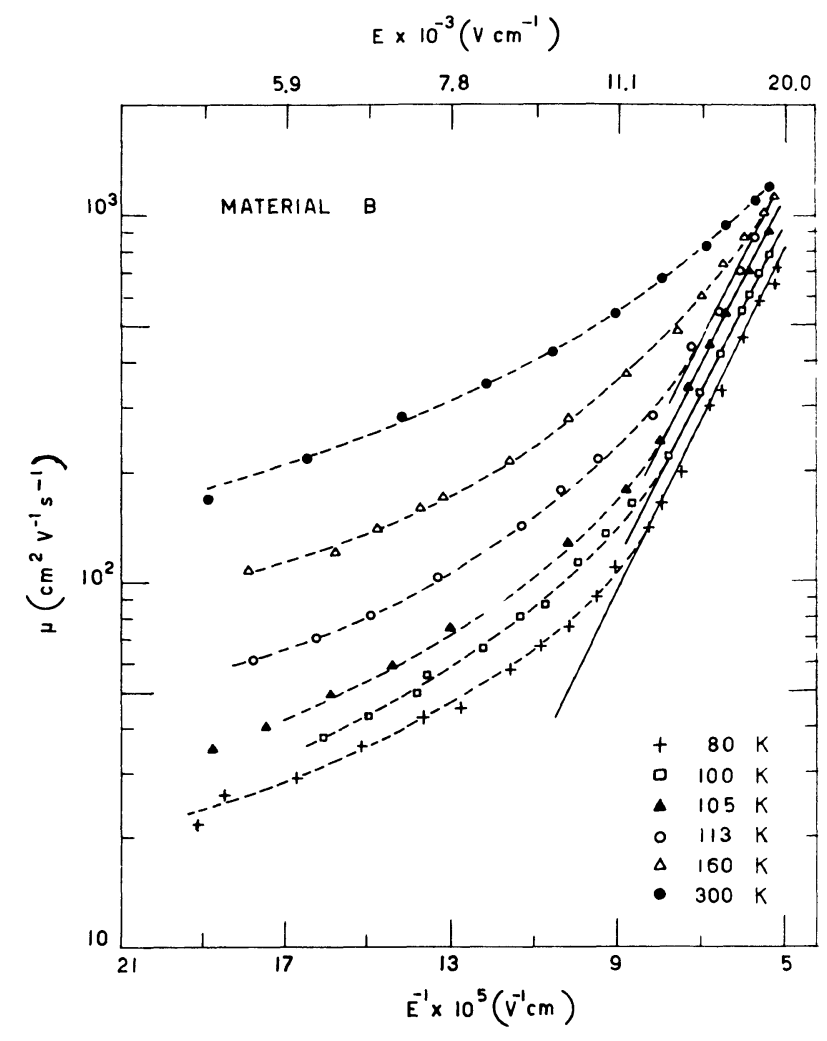

FIG. 11. - Field dependence of electron mobility in CdTe at several temperatures in the high electric field region in $\mathbf{B}$ type materials. The linear variation at high fields of in with $1 / E$ and the temperature dependence of the slope indicate that tunneling is dominating the remission process.

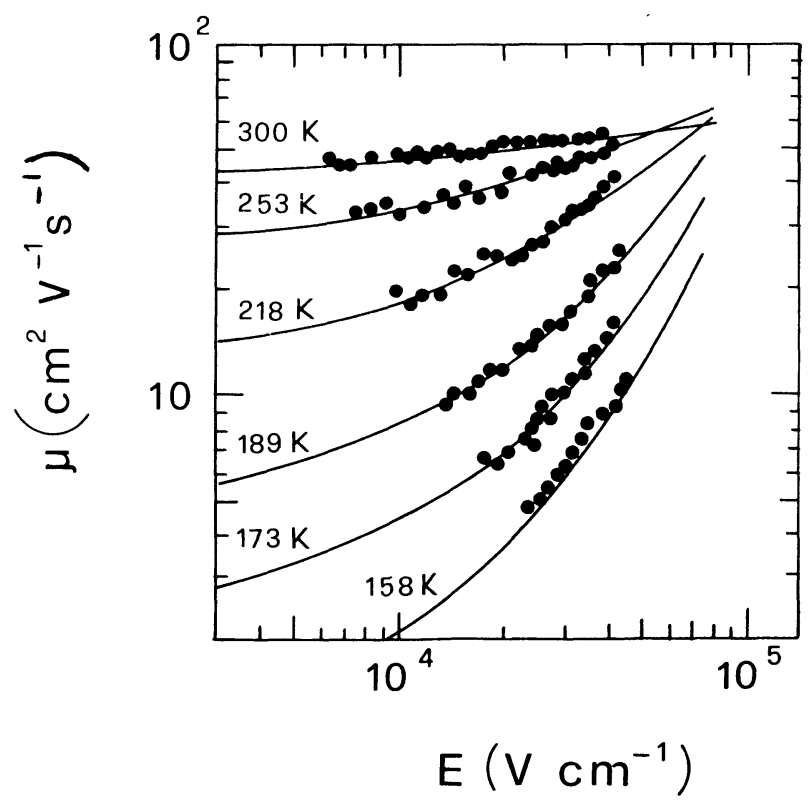

FIg. 12. - Hole mobility in CdTe as a function of electric fields at different temperatures. Full lines show the theoretical results taking into account the Poole-Frenkel effect.

This behaviour, which is not due to the pure lattice scattering, is interpreted as due to trapping and detrapping effect and by the influence on these effects of the 
electric field through the Poole-Frenkel mechanism. The continuous lines in figure 12 are the theoretical results assuming $5 \times 10^{-16} \mathrm{~cm}^{-3}$ trapping centers with a level of $140 \mathrm{meV}^{55}$. The temperature dependence

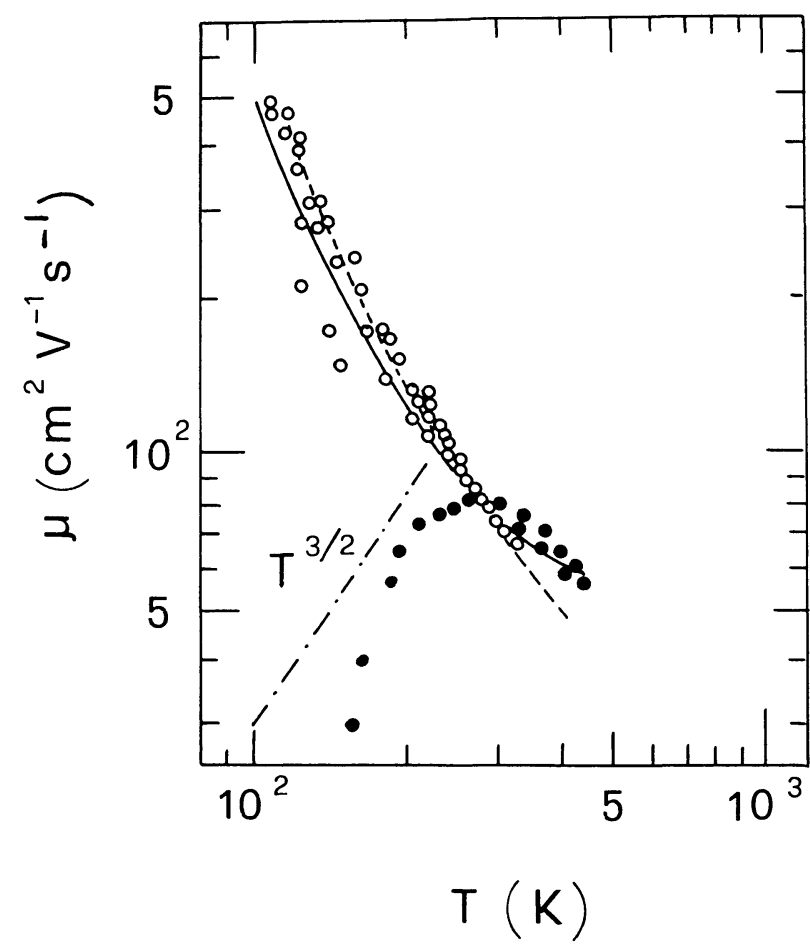

Fig. 13. - Hole mobility in CdTe as a function of temperature. Closed circle refers in our experimental data open circles are from Yamada [17]. The solid line is the theoretical drift mobility obtained with the Monte Carlo calculations (1) while the dashed line came from [17] $\mu=57(\exp .(252 / T)-1)$. of the mobility measured at the lowest applied electric field is reported in figure 13 compared with other experimental data and theoretical expectation considering only lattice scattering mechanism. Only around room temperature the data from high resistivity material approaches the theoretical limit indicating that a considerable number of ionized impurities acting both as traps and as a coulombic scattering center are present.

With TOF technique several trapping levels have been identified. The first was a trap for electrons located at $E_{\mathrm{c}}-0.6 \mathrm{eV}, E_{\mathrm{c}}-0.02$ and $E_{\mathrm{c}}-0.05 \mathrm{eV}$ were successively identified in $\mathrm{In}, \mathrm{Cl}, \mathrm{Br}$ compensated material. Trapping levels for holes were measured at $E_{\mathrm{v}}+0.14$ and $E_{\mathrm{v}}+0.35 \mathrm{eV}$. In the purest material where a $\mu \tau^{+}$product of $10^{-3}-10^{-4} \mathrm{~cm}^{2} \mathrm{~V}^{-1}$ was measured only $E_{\mathrm{c}}-0.02$ and $E_{\mathrm{v}}+0.14 \mathrm{eV}$ have been detected. Such traps are too shallow to be responsible for the values of $\tau^{+}$measured ; in small concentration deeper traps should be present. The elimination of such traps would increase significantly the quality of the material.

4. Conclusion. - The transport properties of electrons and holes in high resistivity compensated CdTe measured with TOF have been revised and compared with theoretical expectations. While the electrons give almost the intrinsic value of mobility for the holes the trapping detrapping phenomena, effects strongly the transport properties.

Several impurity levels have been identified and it is found that traps with energy levels deeper than $0.15 \mathrm{eV}$ should be responsible for the $\mu \tau^{+}$product.

\section{References}

[1] Borsari, V. and Jacoboni, C., Phys. Stat. Sol. (b) 54 (1972) 649.

[2] Stratton, R., Proc. R. Soc. A 246 (1958) 406.

[3] Conwell, E. M., Solid. State Phys. Suppl. 91967.

[4] Costato, M., Jacoboni, C. and Reggiani, L., Phys. Stat. Sol. (b) 52 (1972) 461.

[5] Ottaviani, G., Canali, C., Jacoboni, C., Alberigi Quaranta, A. and Zanio, K. R., J. Appl. Phys. 44 (1973) 360.

[6] Martini, M., MaYer, J. W. and Zanio, K. R. in : Applied Solid State Science Ed. R. Wolfe (Academic Press, New York) 1972, Vol. 3.

[7] Dussel, G. A. and Boer, K. W., Phys. Stat. Sol. 39 (1970) 197.

[8] LAX, M., Phys. Rev. 119 (1960) 1502.

[9] Hearing, R. R., Can. J. Phys. 37 (1959) 1374.
[10] Martini, M. and McMath, T. A., Nucl. Instrum. and Meth. 79 (1969) 259.

[11] Canali, C., Nava, F., Ottaviani, G. and Zanio, K. R., Solid State Commun. 13 (1973) 1255.

[12] Canali, C., Martini, M., Ottaviani, G. and Zanio, K. R., Phys. Rev. B 4 (1971) 422.

[13] Canali, C., Ottaviani, G., Bell, R. O. and Wald, F. V., Phys. Chem. Solids 35 (1974) 1405.

[14] Canal, C., Nava, F., Ottaviani, G. and Zanio, K., Phys. Stat. Sol. (a) 28 (1975) 581.

[15] Shockley, W., Electrons and Holes in Semiconductors (Van Nostrand Co) 1950.

[16] Segall, B., Lorenz, M. and Halsted, R. E., Phys. Rev. 129 (1963) 2471.

[17] Aven, M. and Prener, J. B., Physics and Chemistry of II-VI Compounds (North-Holland, Amsterdam) 1967. 